\title{
Development of an 8-color antibody panel for functional phenotyping of human CD8+ cytotoxic $T$ cells from peripheral blood mononuclear cells
}

\author{
Tara Patel · Amy Cunningham • Martha Holland · John Daley • \\ Suzan Lazo $\cdot$ F. Stephen Hodi $•$ Mariano Severgnini $[$ (I)
}

Received: 20 March 2017/ Accepted: 9 May 2017/Published online: 27 May 2017

(C) Springer Science+Business Media Dordrecht 2017

\begin{abstract}
The study of CD8 positive cells in peripheral blood has become an essential part of research in the field of cancer immunotherapies, vaccine development, inflammation, autoimmune disease, etc. In this study, an 8-color flow cytometry panel, containing lineage and functional markers, was developed for the identification of CD8+ cytotoxic T cells in previously cryopreserved peripheral blood mononuclear cells from healthy human donors. By studying functional markers in naïve and CD3/CD28 activated T cells we demonstrate that the panel is capable of detecting protein markers corresponding to different $\mathrm{T}$ cell activation statuses. Data generated by flow cytometry were corroborated by different antibody based assay technologies to detect soluble cytokines. Our findings suggest that there is an inter donor variability in both baseline and activation responses. We have also successfully developed an antibody panel for flow
\end{abstract}

T. Patel $\cdot$ A. Cunningham - M. Holland .

F. S. Hodi · M. Severgnini $(\bowtie)$

Department of Medical Oncology, Center for Immuno-

Oncology, Dana-Farber Cancer Institute, 450 Brookline,

Ave Jimmy Fund 406, Boston, MA 02215, USA

e-mail: Mariano_severgnini@dfci.harvard.edu

J. Daley $\cdot$ S. Lazo

Department of Medical Oncology/Hematologic

Neoplasia, Dana-Farber Cancer Institute, Boston,

MA 02215, USA cytometry that could be used to study cytotoxic function of $\mathrm{CD} 8 \mathrm{~T}$ cells in clinical immunology research areas.

Keywords CD8+ cytotoxic $\cdot \mathrm{PBMC} \cdot$ Flow cytometry $\cdot$ Antibody panel

\section{Background}

CD8+ T cells are lymphocytes that comprise 5-25\% of total peripheral blood mononuclear cells (PBMCs). They employ a variety of effector mechanisms and are capable of mounting immunogen-specific responses, potentiating immune responses through cytokine release, and enacting cell-mediated killing of virallyinfected and tumor cells through a perforin and granzyme-dependent cytotoxic pathway (Harty et al. 2000; Salti et al. 2011; Pender et al. 2014). The evaluation of specific markers involved in such responses is important to understanding immune response in pathological conditions.

In immune-oncology research, immune phenotyping is critical for evaluating patient responses to clinical immunotherapies and the ability of $\mathrm{T}$ cell based therapies to effectively target tumors (Kalos 2011; Malyguine et al. 2012). Polychromatic flow cytometry assays of CD8 $+\mathrm{T}$ cells compliment currently available immunologic monitoring technologies by enabling more detailed evaluation of 
cytotoxic T lymphocytes and the relationship between effector function and clinical outcomes (Macchia et al. 2013; Zaritskaya et al. 2010). Applications of these assays go beyond the scope of cancer immunotherapies as CD8 $+\mathrm{T}$ cells also play a role in the human immune response to HIV and other viral infections (Kutscher et al. 2008; Speiser et al. 2005).

Using an array of lineage (CD3, CD4, CD8 and CD56), viability dye and functional markers (IFN $\gamma$, MIP1 $\beta$, perforin, and granzyme B) we developed an 8 -color flow cytometry antibody panel for the characterization of $\mathrm{CD} 8+\mathrm{T}$ cells within cryopreserved human PBMC populations isolated from whole blood. This panel allows for the simultaneous evaluation of multiple parameters and offers insight into both the activation status and the cytotoxic function of $\mathrm{CD} 8+\mathrm{T}$ cells.

\section{Materials and methods}

Collection and isolation of healthy donor PBMCs

Leukophoresis samples were obtained from six healthy donors, per the blood collection protocol approved by the Institutional Review Board of Brigham and Women's Hospital. All participants gave written informed consent prior to blood draw. Aphaeresed peripheral blood mononuclear cells (PBMCs) were isolated within $6 \mathrm{~h}$ of blood collection from six healthy donors by density gradient separation with Ficoll-Paque PLUS (GE Healthcare Biosciences, Uppsala, Sweden). Blood was diluted 1:1 with phosphate buffered saline (PBS) and slowly layered on $12 \mathrm{ml}$ of Ficoll-Paque PLUS in a $50 \mathrm{ml}$ conical tube by pipetting down the side of tube with a transfer pipette. Tubes were centrifuged at 436RCFs for 20 min. PBMCs were aspirated from density separation using a transfer pipette, taking precaution not to aspirate Ficoll layers. Cells were washed by adding PBS, followed by centrifugation at 272RCFs for $5 \mathrm{~min}$ and resuspended in fetal bovine serum (FBS; Life Technologies, Carlsbad, CA, USA); $+15 \%$ dimethylsulfoxide (DMSO; Fisher Scientific, Pittsburgh PA, USA) at a concentration of $4-10 \times 10^{7}$ cells $/ \mathrm{ml}$. Cells were frozen overnight at $-80{ }^{\circ} \mathrm{C}$ in $0.5 \mathrm{ml}$ aliquots at a controlled rate of cooling of $-1{ }^{\circ} \mathrm{C} / \mathrm{min}$ and transferred to liquid nitrogen for cryogenic storage until assay.
T cell activation

Frozen PBMCs were thawed in a $37{ }^{\circ} \mathrm{C}$ water bath and resuspended in warmed Dulbecco's Modified Eagle Medium (DMEM) supplemented with 10\% FBS, $1 \times$ antibacterial-antimycotic (Life Technologies). For each stimulation condition, $10 \times 10^{6}$ cells were cultured for $48 \mathrm{~h}$ in six well plates at a concentration of $5 \times 10^{6}$ cells $/ \mathrm{ml}$. Stimulated cells were cultured for 24 or 48 h with $2.6 \times 10^{6} \mathrm{CD} 3 / \mathrm{CD} 28$ Dynabeads/ml (Life Technologies; Trickett and Kwan 2003; Schade et al. 2008). Before staining, Dynabeads were pulled out of the culture using a magnet. Untreated cells (naïve) were used as negative control, and cultured in DMEM $+10 \%$ FBS + antibacterial-antimycotic for $48 \mathrm{~h}$ with out stimulation. No golgi plug, monensin, or brafeldin was added to better mimic PBMCs isolated from patients, as this panel was developed for ex vivo conditions using clinical samples. After culture, cells were harvested and washed by centrifugation at 391RCFs for $5 \mathrm{~min}$ and incubated on ice for the duration of staining procedure. Supernatant from each culture condition was collected by centrifugation and stored undiluted at $-80{ }^{\circ} \mathrm{C}$ until later use in soluble cytokine assays as described below.

\section{Multicolor flow cytometry}

All antibodies and the viability dye were titrated at 0.5 , 1 and $2 X$ the recommended concentrations. Both naïve and activated cells were tested, and optimal concentrations were determined by choosing the lowest concentration where positive populations were clearly visible in FlowJo. The concentration of Zombie NIR is undisclosed by vendor for proprietary reasons. Instead, the volume of dye was resuspended in $100 \mu \mathrm{L}$ DMSO (stock solution).

Cells were resuspended at $6.7 \times 10^{6}$ cells $/ \mathrm{ml}$ in PBS and aliquoted in a 96 well plate at $1 \times 10^{6}$ cells/ well. After washing twice with PBS to remove medium, cells were stained with Zombie NIR fixable viability dye at a 1:2500 dilution of the stock solution into PBS in a total volume of $150 \mu \mathrm{l}$. Cells were incubated for $15 \mathrm{~min}$ on ice and then centrifuged at 272RCFs for $5 \mathrm{~min}$ at $4{ }^{\circ} \mathrm{C}$ (all centrifugations for the staining procedure were performed under these conditions). Cells were washed once with $150 \mu \mathrm{l}$ PBS and then incubated on ice for 18 min in Fc Block (Miltenyi Biotec, Cambridge, MA, USA), diluted 1:625 in FACS 
buffer (PBS, 2.5\% FBS). After Fc block incubation, cells were washed once with FACS buffer and stained for surface markers CD3-PeCy7, CD8-vioBright FITC and CD56-PerCPcy5.5 in $150 \mu \mathrm{l}$ total volume for 45 min, diluted per titration results (Table 2). Cells were then washed three times in $150 \mu$ FACS buffer and immediately fixed and permeabilized with BD Cytofix/Cytoperm per manufacturer's protocol (BD Biosciences, San Jose, CA, USA). After fixation, cells were stained for intracellular markers perforin-PE, MIP1 $\beta$-BV421, IFN $\gamma$ BV510, and granzyme B-AF647 in $150 \mu \mathrm{l}$ of BD perm/wash buffer, following manufacturer's instructions, at the concentrations reported in Table 2. Antibody clones, fluorophores, vendors and catalog numbers are provided in Table 1.

Cells were acquired using an LSRII analyzer (BD Biosciences) with the laser configuration described in Table 2. Daily maintenance was run on LSRII in which freshly prepared ( $\sim 2$ drops $/ 0.5 \mathrm{ml}$ in $\mathrm{H}_{2} \mathrm{O}$ ) eight peak rainbow beads were acquired and recorded for 10,000 scatter gated events every morning after thorough cleaning (5-10 min) the instruments with $10 \%$ bleach and distilled water. Bead peak 7 was standardized to be centered at a 50,000-peak channel number for all detectors and the relative median fluorescence intensity on the all detectors excited by each laser was recorded

Table 1 Information about antibodies and viability dye used for the development of the CD8+ multicolor flow cytometry panel

\begin{tabular}{llllll}
\hline Specificity & Clone & Fluorophore & Vendor & Catalog number & Purpose \\
\hline CD3 & UCHT1 & PE-Cy7 & eBioscience (San Diego, CA, USA) & $25-0038-42$ & Lineage \\
CD8 & BW135/80 & vioBright FITC & Myltenyi Biotec & $130-104-519$ & Lineage \\
CD56 & B159 & PerCPcy5.5 & BD Biosciences & 560842 & Lineage (negative gate) \\
Perforin & BD48 & PE & Biolegend (San Diego, CA, USA) & 353303 & Cytotoxic function \\
MIP1 $\beta$ & D21-1351 & BV421 & BD Biosciences & 562900 & Activator \\
IFN $\gamma$ & b27 & BV510 & BD Biosciences & 563287 & Cytotoxic function \\
Granzyme B & GB11 & AF647 & BD Biosciences & 560212 & Cytotoxic function \\
Viability dye & - & Zombie NIR & Biolegend & 423105 & Dead cell exclusion \\
\hline
\end{tabular}

Table 2 LSRII flow cytometer bandpass and specific emission filters utilized for each fluorophore for the configuration of LSRII laser lines

\begin{tabular}{|c|c|c|c|c|c|c|c|c|}
\hline Laser line & $488 \mathrm{~nm}$ & & & & $633 \mathrm{~nm}$ & & $407 \mathrm{~nm}$ & \\
\hline Emission filters & $530 / 30$ & $575 / 26$ & $710 / 50$ & $780 / 60$ & $660 / 20$ & $780 / 60$ & $450 / 50$ & $525 / 50$ \\
\hline Bandpass & 505 & 550 & & 755 & & 755 & & 505 \\
\hline Fluorochrome & AF488 & PE & PerCPcy5.5 & PEcy7 & AF647 & Zombie NIR & BV421 & BV510 \\
\hline
\end{tabular}

Fig. 1 Sample gating strategy. FlowJo generated Contour plots (left) and dot plots (right) were used to determine gates. FMO and unstimulated cells were used as baseline
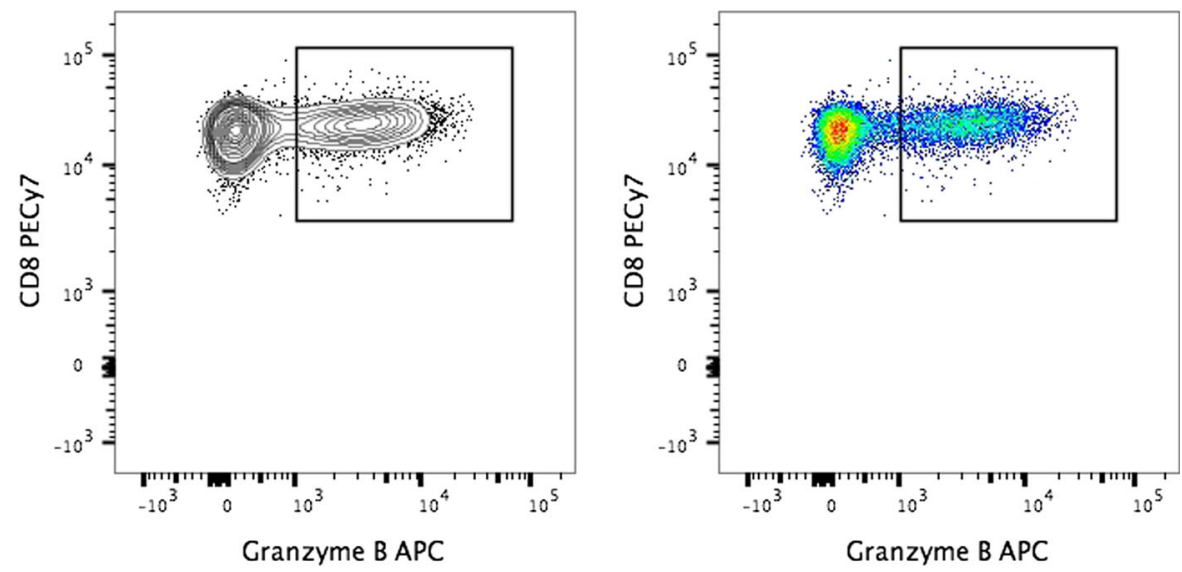

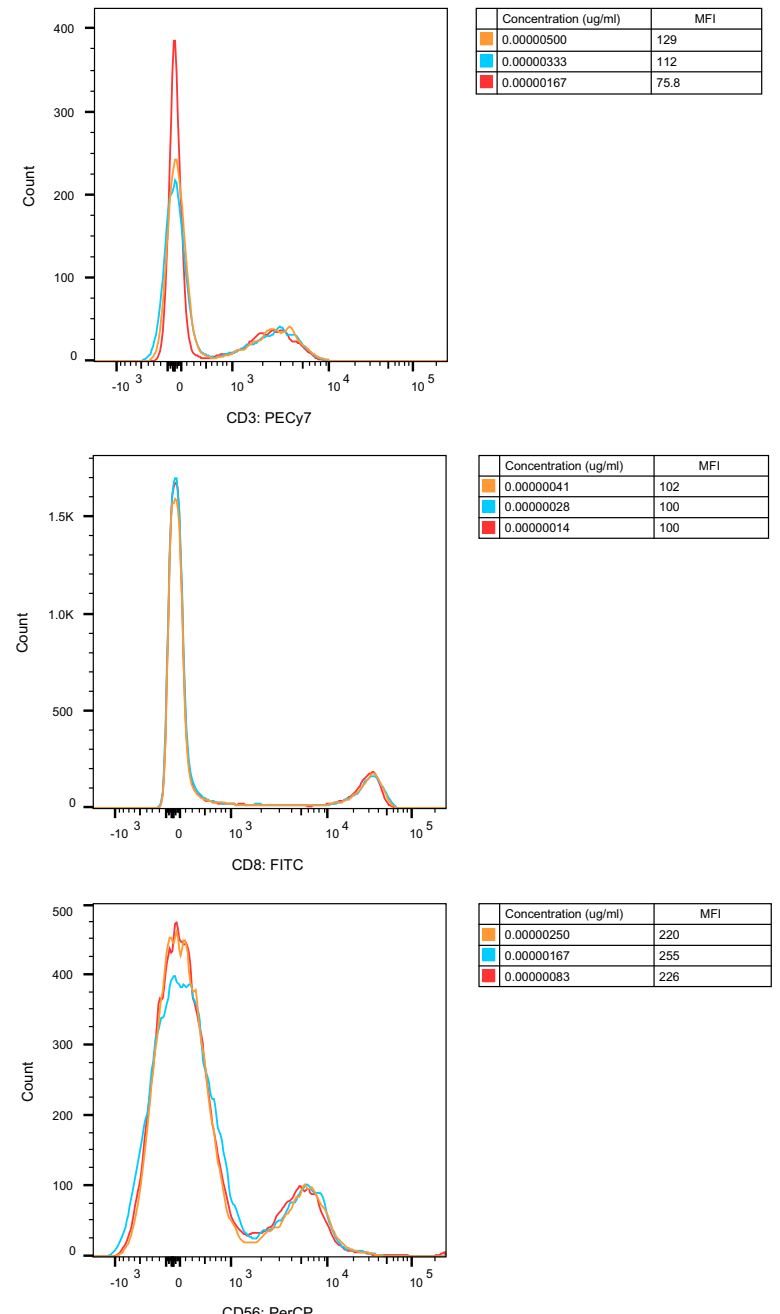

226


0.00000005
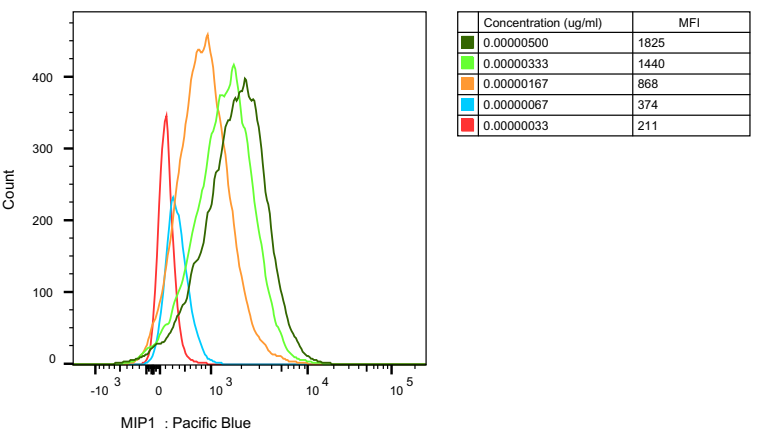

each marker at different concentrations and their median fluorescent intensities (MFIs). Keys represent antibody and viability dye concentrations tested and corresponding MFIs

Compensation controls were established using pooled cells from all donors. A mean of 80,000 events was collected for each control and experimental population. 
Table 3 Final

concentration of antibody (Ab) conjugates added to the panel and stock concentration from the manufacturers

\begin{tabular}{llll}
\hline Specificity & Fluorophore & $\begin{array}{l}\text { Ab amount per well } \\
(\mu \mathrm{g})\end{array}$ & $\begin{array}{l}\text { Stock Ab concentration } \\
(\mu \mathrm{g} / \mathrm{ml})\end{array}$ \\
\hline CD3 & PeCy7 & 0.5 & 100 \\
CD8 & vioBright FITC & 0.0206 & 8.25 \\
CD56 & PerCPCy5.5 & 0.25 & 50 \\
Perforin & PE & 0.0075 & 3 \\
MIP1 $\beta$ & BV421 & 0.1 & 100 \\
IFN $\gamma$ & BV510 & 0.5 & 100 \\
Granzyme B & AF647 & 0.025 & 10 \\
\hline
\end{tabular}

Table 4 Voltages set on LSRII for each parameter applied to the antibody panel to keep cell populations within scale and obtain the best separation of negative and positive cell populations

\begin{tabular}{ll}
\hline Parameter & Voltage \\
\hline FSC-A & 423 \\
FSC-H & 423 \\
FSC-W & 423 \\
SSC-A & 374 \\
SSC-H & 374 \\
SSC-W & 374 \\
FITC-A & 551 \\
PE-A & 545 \\
PerCPCy5.5-A & 769 \\
PE-Cy7-A & 621 \\
APC-A & 467 \\
APC-Cy7-A & 687 \\
AmCyan-A & 368 \\
Pacific Blue-A & 382 \\
\hline
\end{tabular}

Electronic FCS files were analyzed and gated using FlowJo software (Treestar, Ashland, OR, USA). Gating strategy was determined by fluorescence minus one (FMO) controls for granzyme B, perforin, MIP1 $\beta$, and IFN $\gamma$ and contour plots (Fig. 1).
Natural Killers (CD3-CD56+) and Natural Killer $T$ cells $(\mathrm{CD} 3+\mathrm{CD} 56+)$ were excluded from intracellular cytokine analysis (Grossman et al. 2004).

Bar graphics were generated in Graphpad Prism version 7 (GraphPad Software, La Jolla, CA, USA).

Soluble biomarker detection

Cell culture supernatant was diluted 1:4 and used for perforin ELISA (Abcam, Cambridge, MA, USA), and for a 3-plex cytokine assay of IFN $\gamma$, granzyme B, and MIP1 $\beta$ (Affymetrix, Santa Clara, CA, USA) following manufacturers protocol and read on a Luminex MAGPIX Analyzer (Bio-Rad, Hercules, CA, USA).

\section{Results}

All antibodies and viability dye were titrated and the concentrations that gave the best separation between negative and positive populations were selected to use in the panel as shown in MFIs representing histograms (Fig. 2).

Table 5 Average compensation of three technical replicates of each fluorophore applied to the antibody panel

\begin{tabular}{lllllllll}
\hline & FITC-A & PE-A & PerCPCy5.5-A & PE-Cy7-A & APC-A & APC-Cy7-A & AmCyan-A & Pacific blue-A \\
\hline FITC-A & 1.00 & 0.30 & 0.03 & 0.00 & 0.00 & 0.00 & 0.01 & 0.00 \\
PE-A & 0.01 & 1.00 & 0.13 & 0.01 & 0.00 & 0.00 & 0.00 & 0.00 \\
PerCP-Cy5-5-A & 0.01 & 0.02 & 1.00 & 0.11 & 0.00 & 0.03 & 0.01 & 0.01 \\
PE-Cy7 & 0.00 & 0.04 & 0.03 & 1.00 & 0.00 & 0.05 & 0.00 & 0.00 \\
APC-A & 0.01 & 0.00 & 0.07 & 0.00 & 1.00 & 1.05 & 0.01 & 0.01 \\
APC-Cy7-A & 0.01 & 0.00 & 0.13 & 0.35 & 0.00 & 1.00 & 0.01 & 0.01 \\
AmCyan-A & 0.05 & 0.05 & 0.05 & 0.01 & 0.00 & 0.01 & 1.00 & 0.47 \\
Pacific blue-A & 0.00 & 0.00 & 0.00 & 0.00 & 0.00 & 0.00 & 0.04 & 1.00 \\
\hline
\end{tabular}



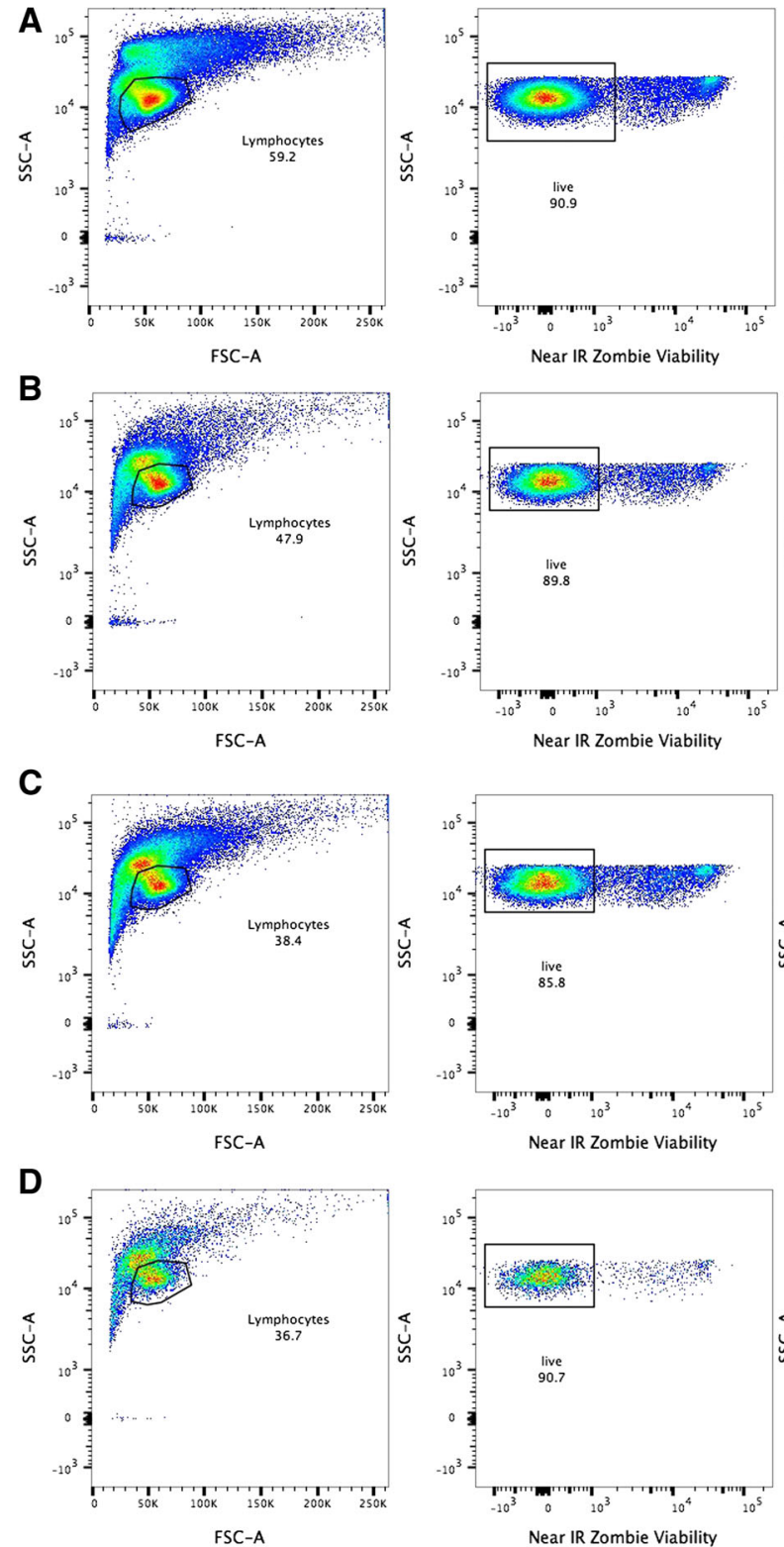
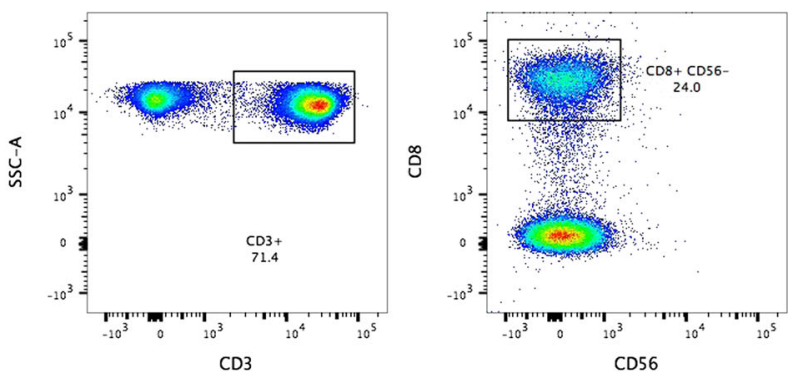

$\mathrm{CD} 3$
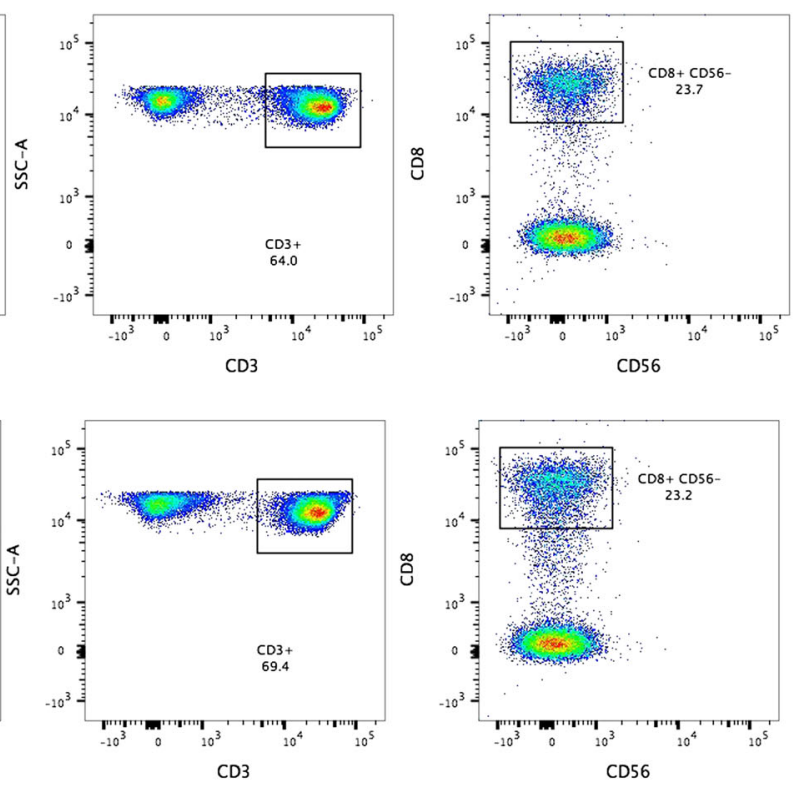

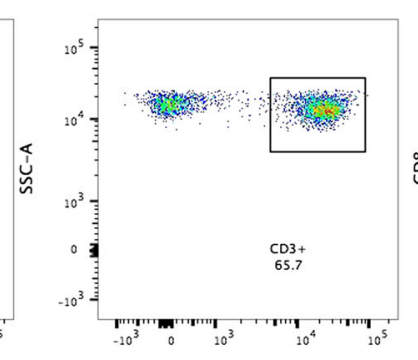

CD3

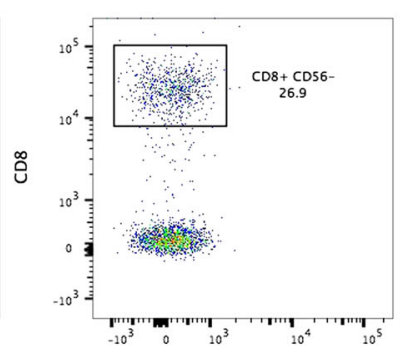

CD56

Fig. 3 FlowJo dot plots demonstrating gating strategy for the lineage markers in untreated, cultured (A), $24 \mathrm{~h}$ stimulation (B), $48 \mathrm{~h}$ stimulation (C) and the FMO control for functional markers (D). All cells were gated for Lymphocytes/Live Cells/CD3+/CD8+/CD56

After the appropriate concentration of antibodies was established, the same amounts of antibodies and viability dye were used in the panel consistently across all donors (Table 3).

Voltages were set such that the negative population fell at approximately $10^{3}$ MFIs and the positive population was on scale, and voltages were held constant between samples with different concentrations of the same antibody to ensure that the changes in
MFIs were due to antibody concentration and not voltage (Table 4).

Compensation was applied to the panel to account for spillover between the channels (Table 5).

Gating plots for Lymphocytes/live/CD3+/CD8+, CD56- cells were done based on FMOs and unstained controls where a good separation of the parent populations was observed (Fig. 3). The gating strategy to detect functional markers was performed as shown 

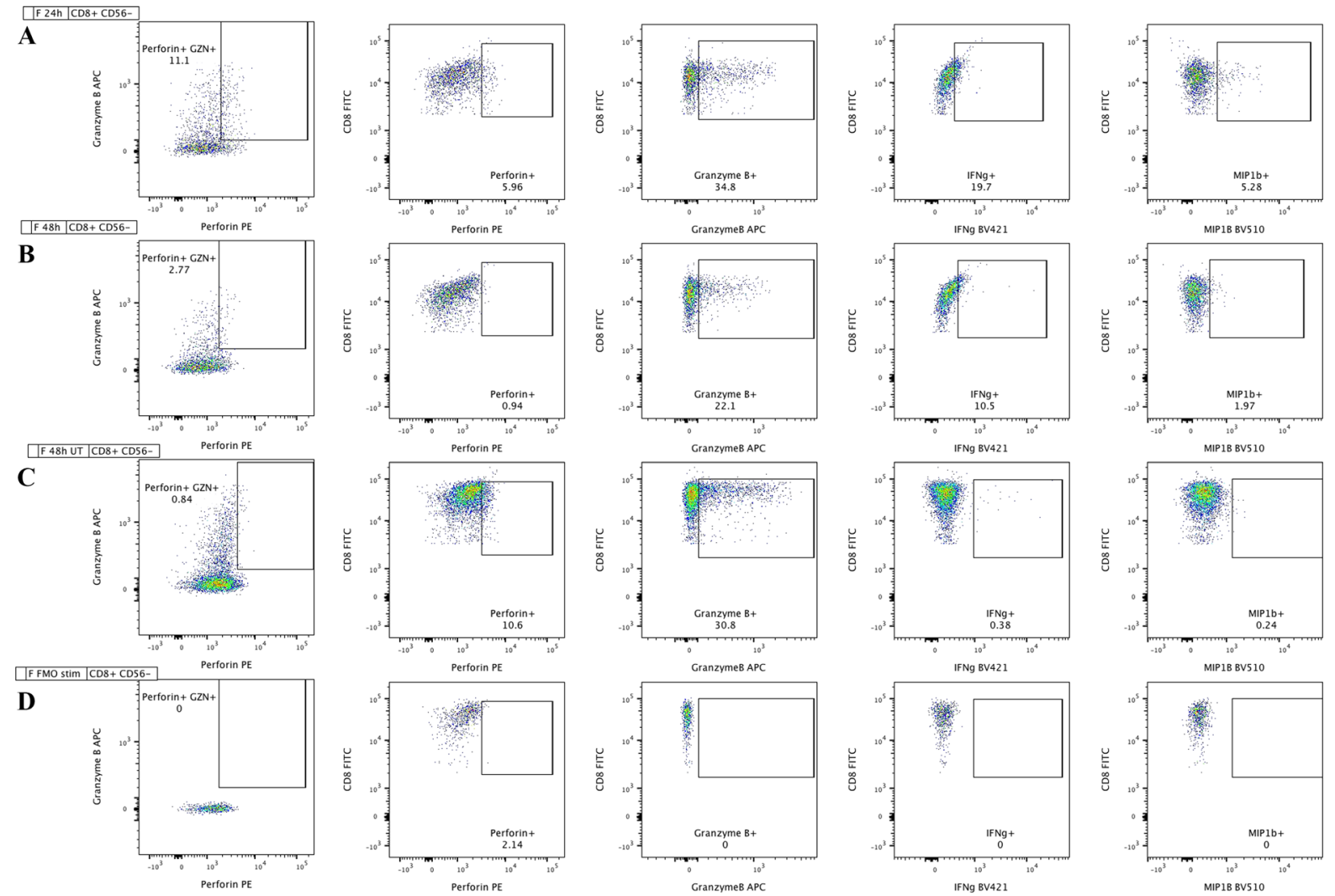

Fig. 4 Gating strategy for CD8 + cells expressing functional markers. Dot plots show lymphocytes/single cells/live/CD3+/ CD8+/CD56- population of cells from untreated, cultured (A),

in Fig. 4 where the double positive populations $(\mathrm{CD} 8+/ \mathrm{MIP} 1 \beta+, \quad \mathrm{CD} 8+/$ perforin,$+ \quad \mathrm{CD} 8+/$ granzyme $\mathrm{B}+$ and $\mathrm{CD} 8+/ \mathrm{IFN} \gamma+$ ) were defined per FMOs and unstained controls. All gatings were performed individually by sample.

Figure 5 represents averages of percent cellular populations of technical replicates of all PBMCs donors. $\mathrm{CD} 3+$ and $\mathrm{CD} 8+$ populations do not seem to change within the same donor depending on stimulation status (Fig. 4A, B). The functional markers show an increasing trend in percent of double positive populations (Fig. 4C-F). We observed an inter donor variation in unstimulated parent populations that did not necessarily translate to stimulated populations (e.g. Donor E). Fold changes were calculated for each single donor, marker and stimulation treatment (Table 6). We show increases in functional markers between 1.2- and 100-fold in stimulated cells depending on each donor and stimulation time point.
$24 \mathrm{~h}$ stimulation (B), $48 \mathrm{~h}$ stimulation $(\mathbf{C})$ and the FMO control (D). Shown here are representative data, using Donor A

The concentrations of secreted proteins perforin, granzyme B, IFN $\gamma$ and MIP1 $\beta$ increased from 24 to $48 \mathrm{~h}$ across the donor population, except for MIP1 $\beta$ in Donor B. IFN $\gamma$ was notdetected in the untreated cell culture supernatant of Donors C, D, and F (Fig. 6). Increased levels of intracellular markers were also observed by soluble protein detection assays for the same proteins.

\section{Discussion}

It is advantageous to use a flow cytometry antibody panel that encompasses multiple functional markers simultaneously to more completely understand the activity of cytotoxic cells. Multiparametric flow allows for the same cells to be evaluated in a multidimensional capacity and gives insight into the activity of the cells of interest. Unlike past studies 
Fig. 5 Summary data from three iterations of the flow cytometry panel. All data are percent of parent population. All cells were gated lymphocytes/live/ singlets/CD3+. Averaged granzyme $\mathbf{B}+(\mathbf{A})$, perforin $+(\mathbf{B}), \operatorname{MIP} 1 \beta+(\mathbf{C})$, IFN $\gamma+(\mathbf{D})$ and granzyme + / perforin $+(\mathbf{E})$, data with SEM. Data were analyzed for general patterns of change and no statistical tests were run
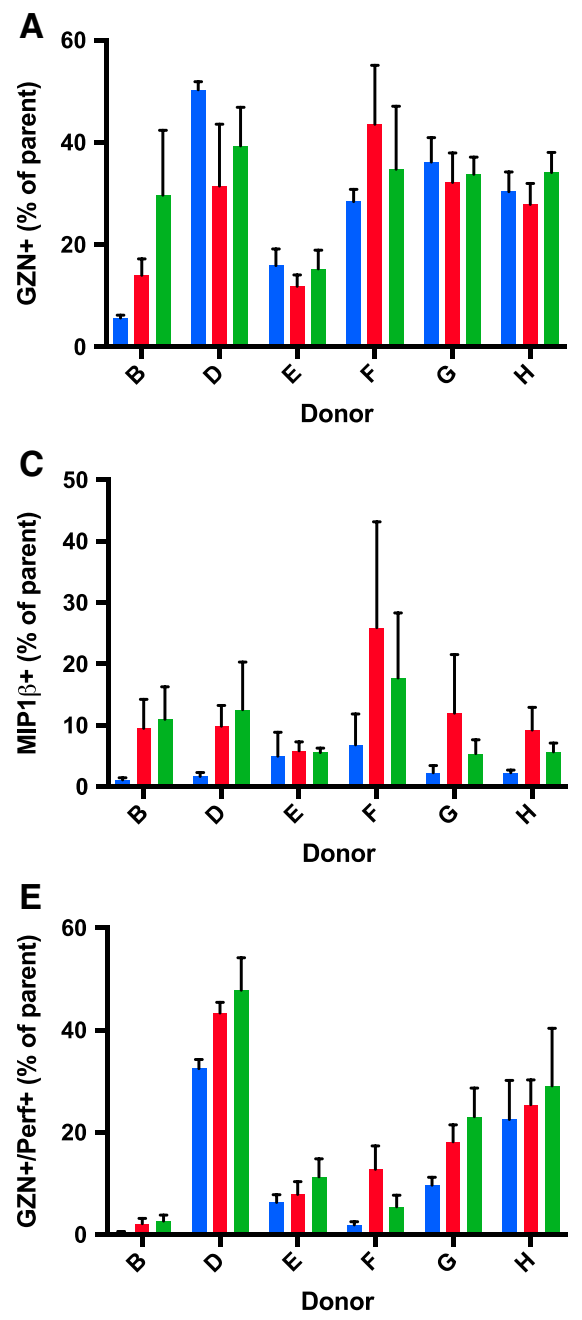
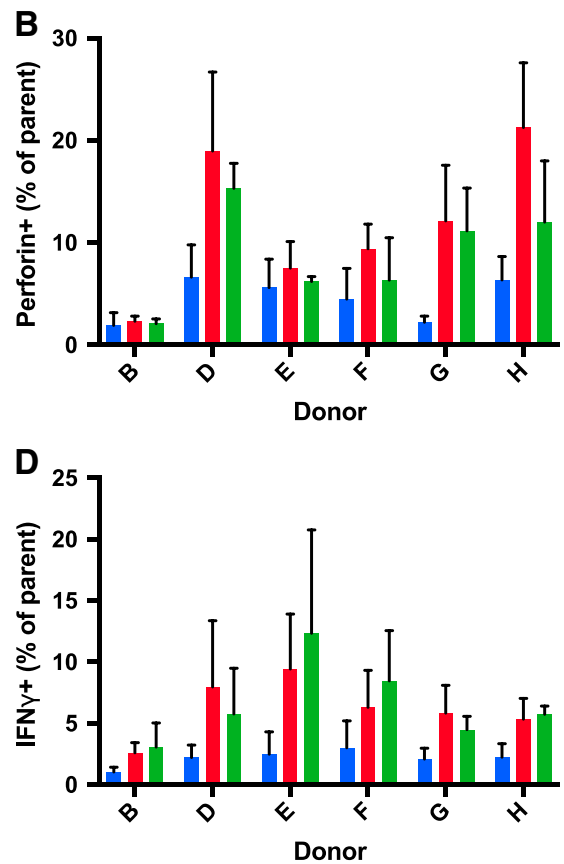

Table 6 Fold differences from percent populations of 24 and $48 \mathrm{~h}$ stimulated PBMCs (24 and $48 \mathrm{~h}$ ) from six healthy donors when compared to unstimulated cells

\begin{tabular}{llllcrr}
\hline & CD3+ & CD8 & Perforin + & Granzyme B + & IFN $\gamma$ & MIP1 $\beta+$ \\
\hline Donor A 24 h & 0.75 & 0.79 & 2.88 & 6.42 & 9.93 & 140.02 \\
Donor A 48 h & 0.80 & 0.80 & 1.98 & 12.16 & 6.88 & 139.58 \\
Donor B 24 h & 0.95 & 0.67 & 1.98 & 1.62 & 1.33 & 94.38 \\
Donor B 48 h & 1.27 & 0.77 & 1.97 & 1.68 & 0.80 & 36.06 \\
Donor C 24 h & 1.04 & 1.06 & 1.99 & 1.20 & 13.52 & 50.67 \\
Donor C 48 h & 1.29 & 0.85 & 1.58 & 2.39 & 10.22 & 23.75 \\
Donor D 24 h & 0.93 & 0.75 & 5.28 & 1.83 & 2.21 & 155.51 \\
Donor D 48 h & 0.77 & 0.89 & 4.48 & 0.96 & 2.57 & 51.02 \\
Donor E 24 h & 0.76 & 1.00 & 20.15 & 1.80 & 5.76 & 43.69 \\
Donor E 48 h & 0.88 & 1.02 & 17.94 & 1.50 & 4.28 & 19.33 \\
Donor F 24 h & 0.92 & 0.83 & 1.84 & 1.01 & 3.37 & 27.45 \\
Donor F 48 h & 0.98 & 0.83 & 1.18 & 0.86 & 3.47 & 19.33 \\
\hline
\end{tabular}



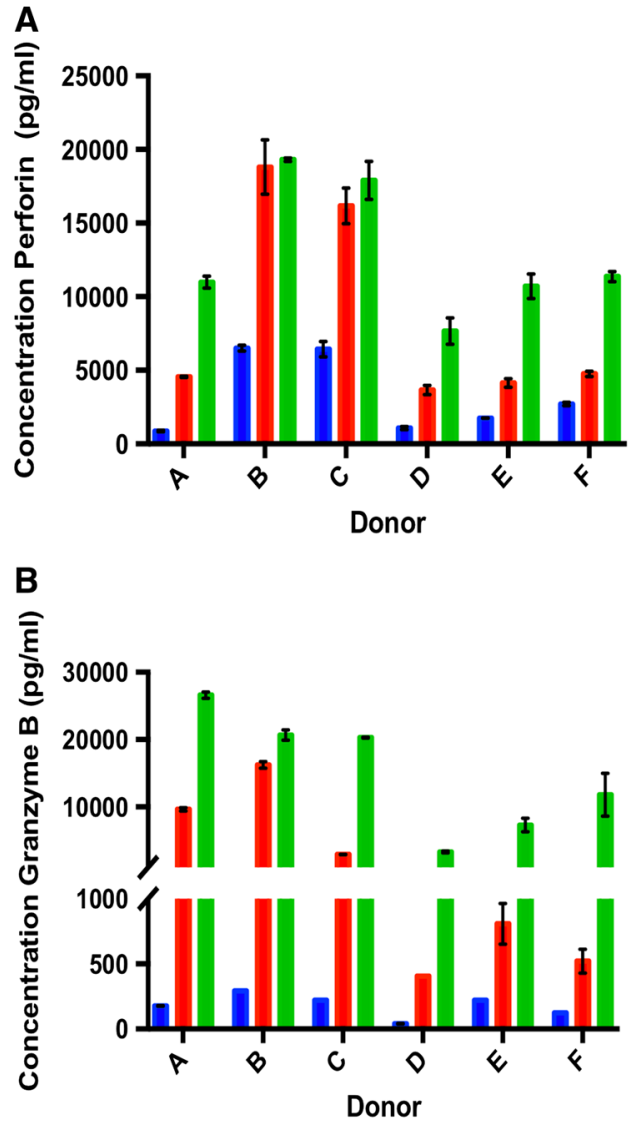

Fig. 6 Soluble protein markers in healthy donors. Bar graphs reflect the mean observed concentrations, measured by ELISA, of perforin (A), and by multiplex assay of granzyme B (B), IFN $\gamma$ (C), and MIP1 $\beta$ (D) from two technical replicates from

(Horton et al. 2007), this panel development uses T-cell specific activation and analyzes cells in a context specific to immune oncology by including perforin and granzyme in the analysis.

Before the panel development, it is essential that all antibodies and viability dyes are titrated to the right concentration where positive cell populations can be distinguished from negative ones. As previously described, CD3/CD28 Dynabeads can activate human T cells (Trickett and Kwan 2003; Schade et al. 2008). Here, in the in vitro stimulation with Dynabeads, we have demonstrated the ability of the panel to distinguish between activated and non-activated subsets of CD8+ cells by measuring cell populations expressing IFN $\gamma$, MIP1 $\beta$, perforin, and granzyme B. By studying functional markers in unstimulated and activated $\mathrm{T}$ cells populations, we demonstrate that the panel can detect various levels of protein expression that may

D
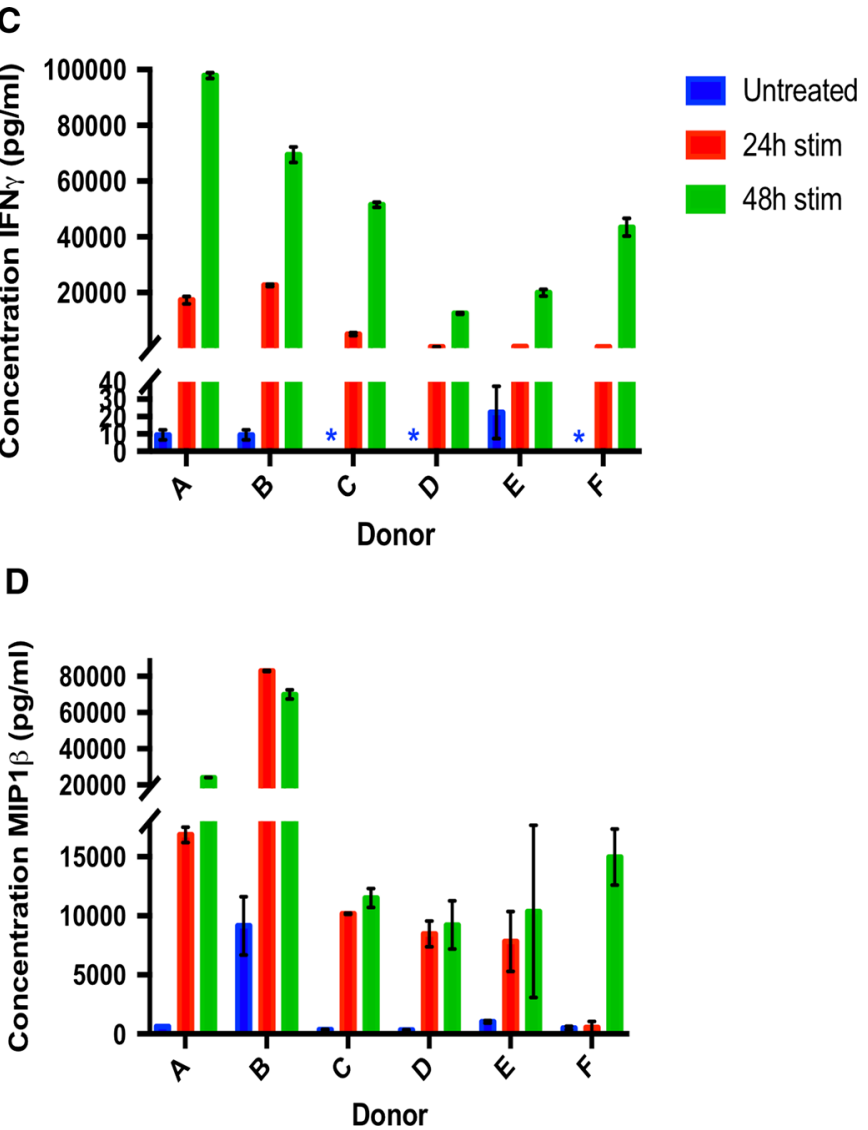

unstimulated (untreated), and stimulated cells (24 and $48 \mathrm{~h}$ stimulation with CD3/CD28 Dynabeads). *Designates samples at undetectable concentrations

occur in vivo. Cytokine expression levels and degree of response to Dynabead activation can vary as seen in these results due to many uncontrolable variables. These data may differ based on the race, gender, age and other factors, as seen previously (Swee et al. 2016; Koide and Engleman 1990; Bernin et al. 2016; Bouman et al. 2004; Kee et al. 2012; Laux et al. 2000; Nociari et al. 1999). Thus, inter-donor variation in baseline expression levels, health status and individual response to $\mathrm{CD} 3 / \mathrm{CD} 28$ activation is normal, and due to the anonymity of the donors was not controlled for.

The soluble biomarker data shown here is a functional validation of cytotoxic $\mathrm{T}$ cells ability to release intracellular cytokines and enzymes assessed in this panel. Since supernatant was collected from total PBMC culture, it is impossible to determine the specific cell subtypes responsible for generating the 
cytokines and enzymes detected in the multiplex and ELISA results from cell culture supernatants. Whereas supernatants contained accumulated soluble proteins released, proteins detected in flow cytometry were expressed by the cells at the time when the assay was performed. Thus, these data should not be used in place of one another but rather as complimentary technologies to better understand the functional status of CD8+ $\mathrm{T}$ cells in an in vitro system.

CD8+ cytotoxic $\mathrm{T}$ cells play a role in immune response to cancers, autoimmune diseases, inflammation and viral infections including HIV (Di Meglio and Duarte 2013; Salti et al. 2011; Speiser et al. 2005). For example, in the context of lung and melanoma cancer research, exploration of activated CD8 $+\mathrm{T}$ cells is of interest in studying tumor evasion of perforin and granzyme-mediated killing (Hodge et al. 2014; Daud et al. 2016). It is of further interest to study CD8+ T cells within tumor-infiltrating lymphocyte populations to explore effector molecules released by activated $\mathrm{CD} 8+\mathrm{T}$ cells that are recruited to the tumor microenvironment in the context of various cancer therapies (Oelkrug and Ramage 2014).

\section{Conclusions}

In this study, we show the development of an 8-parameter flow cytometry antibody panel that identifies cytotoxic CD8+ $\mathrm{T}$ cells, and their functional status, using cryopreserved PBMCs from healthy donors, and could potentially be applied to any area of clinical research where $\mathrm{CD} 8+$ cells play a role as a cellular biomarker.

\section{References}

Bernin H, Fehling H, Marggraff C, Tannich E, Lotter H (2016) The cytokine profile of human NKT cells and PBMCs is dependent on donor sex and stimulus. Med Microbiol Immunol 205:321-332

Bouman A, Schipper M, Heineman MJ, Faas MM (2004) Gender difference in the non-specific and specific immune response in humans. Am J Reprod Immunol 52:19-26

Daud AI, Loo K, Pauli ML, Sanchez-Rodriguez R, Sandoval PM, Taravati K, Tsai K, Nosrati A, Nardo L, Alvarado MD, Algazi AP, Pampaloni MH, Lobach IV, Hwang J, Pierce RH, Gratz IK, Krummel MF, Rosenblum MD (2016) Tumor immune profiling predicts response to anti-PD-1 therapy in human melanoma. J Clin Invest 126:3447-3452
Di Meglio P, Duarte JH (2013) CD8 T cells and IFN- $\gamma$ emerge as critical players for psoriasis in a novel model of mouse psoriasiform skin inflammation. J Invest Dermatol 133:871-874

Grossman WJ, Verbsky JW, Tollefsen BL, Kemper C, Atkinson JP, Ley TJ (2004) Differential expression of granzymes A and $\mathrm{B}$ in human cytotoxic lymphocyte subsets and $\mathrm{T}$ regulatory cells. Blood 104:2840-2848

Harty JT, Tvinnereim AR, White DW (2000) CD8+ T cell effector mechanisms in resistance to infection. Annu Rev Immunol 18:275-308

Hodge G, Barnawi J, Jurisevic C, Moffat D, Holmes M, Reynolds PN, Jersmann H, Hodge S (2014) Lung cancer is associated with decreased expression of perforin, granzyme B and interferon (IFN)-gamma by infiltrating lung tissue T cells, natural killer (NK) T-like and NK cells. Clin Exp Immunol 178:79-85

Horton H, Thomas EP, Stucky JA, Frank I, Moodie Z, Huang Y, Chiu YL, McElrath MJ, De Rosa SC (2007) Optimization and validation of an 8-color intracellular cytokine staining (ICS) assay to quantify antigen-specific T cells induced by vaccination. J Immunol Methods 323:39-54

Kalos M (2011) Biomarkers in T cell therapy clinical trials. J Transl Med 9:138-147

Kee SJ, Park YW, Cho YN, Jin HM, Kim MJ, Lee SJ, Kim TJ, Lee SS, Kwon YS, Jang HC, Kim N, Shin MG, Shin JH, Suh SP, Ryang DW (2012) Age-and gender-related differences in circulating natural killer T cells and their subset levels in healthy Korean adults. Hum Immunol 73:1011-1016

Koide J, Engleman EG (1990) Differences in surface phenotype and mechanism of action between alloantigen-specific CD8+ cytotoxic and suppressor T cell clones. J Immunol 144:32-40

Kutscher S, Dembek CJ, Allgayer S, Heltai S, Stadlbauer B, Biswas P, Nozza S, Tambussi G, Bogner JR, Stellbrink HJ, Goebel FD, Lusso P, Tinelli M, Poli G, Erfle V, Pohla H, Malnati M, Cosma A (2008) The intracellular detection of MIP-1Beta enhances the capacity to detect IFN-gamma mediated HIV-1-specific CD8 T-cell responses in a flow cytometric setting providing a sensitive alternative to the ELISPOT. AIDS Res Ther 6:5-22

Laux I, Khoshnan A, Tindell C, Bae D, Zhu X, June CH, Effros RB, Nel A (2000) Response differences between human CD4+ and CD8+ T-cells during CD28 costimulation: implications for immune cell-based therapies and studies related to the expansion of double-positive T-cells during aging. Clin Immunol 96:187-197

Macchia I, Urbani F, Proietti E (2013) Immune monitoring in cancer vaccine clinical trials: critical issues of functional flow cytometry-based assays. Biomed Res Int 2013:726239

Malyguine AM, Strobl S, Dunham K, Shurin MR, Sayers TJ (2012) ELISPOT assay for monitoring cytotoxic T lymphocytes (CTL) activity in cancer vaccine clinical trials. Cells 1:111-126

Nociari MM, Telford W, Russo C (1999) Postthymic development of CD28- CD8+ T cell subset: age-associated expansion and shift from memory to naive phenotype. J Immunol 162:3327-3335

Oelkrug C, Ramage JM (2014) Enhancement of T cell recruitment and infiltration into tumors. Clin Exp Immunol 178:1-8 
Pender MP, Csurhes PA, Pfluger CM, Burrows SR (2014) Deficiency of CD8+ effector memory $\mathrm{T}$ cells is an early and persistent feature of multiple sclerosis. Mult Scler 20:1825-1832

Salti SM, Hammelev EM, Grewal JL, Reddy ST, Zemple SJ, Grossman WJ, Grayson MH, Verbsky JW (2011) Granzyme B regulates antiviral CD8+ T cell responses. J Immunol 187:6301-6309

Schade AE, Schieven GL, Townsend R, Jankowska AM, Susulic V, Zhang R, Szpurka H, Maciejewski JP (2008) Dasatinib, a small-molecule protein tyrosine kinase inhibitor, inhibits T-cell activation and proliferation. Blood 111:1366-1377

Speiser DE, Liénard D, Rufer N, Rubio-Godoy V, Rimoldi D, Lejeune F, Krieg AM, Cerottini JC, Romero P (2005)
Rapid and strong human CD8 + T cell responses to vaccination with peptide, IFA, and $\mathrm{CpG}$ oligodeoxynucleotide 7909. J Clin Invest 115:739-746

Swee LK, Tan ZW, Sanecka A, Yoshida N, Patel H, Grotenbreg G, Frickel EM, Ploegh HL (2016) Peripheral self-reactivity regulates antigen-specific CD8 T-cell responses and cell division under physiological conditions. Open Biol 6:160293

Trickett A, Kwan YL (2003) T cell stimulation and expansion using anti-CD3/CD28 beads. J Immunol Methods 275:251-255

Zaritskaya L, Shurin MR, Sayers TJ, Malyguine AM (2010) New flow cytometric assays for monitoring cell-mediated cytotoxicity. Expert Rev Vaccines 9:601-616 